\title{
What is indentation hardness?
}

\author{
Yang-Tse Cheng ${ }^{\mathrm{a}, *}$, Che-Min Cheng ${ }^{\mathrm{b}}$ \\ ${ }^{\mathrm{a}}$ Materials and Processes Laboratory, General Motors Research and Development Center, Warren, MI 48090, USA \\ ${ }^{\mathrm{b}}$ Laboratory for Non-Linear Mechanics of Continuous Media, Institute of Mechanics, Chinese Academy of Sciences, Beijing 100080, \\ PR China
}

\begin{abstract}
Using dimensional analysis and finite element calculations, we derive simple scaling relationships for loading and unloading curve, contact depth, and hardness. The relationship between hardness and the basic mechanical properties of solids, such as Young's modulus, initial yield strength, and work-hardening exponent, is then obtained. The conditions for 'piling-up' and 'sinking-in' of surface profiles during indentation are determined. A method for estimating contact depth from initial unloading slope is examined. The work done during indentation is also studied. A relationship between the ratio of hardness to elastic modulus and the ratio of irreversible work to total work is discovered. This relationship offers a new method for obtaining hardness and elastic modulus. Finally, a scaling theory for indentation in power-law creep solids using self-similar indenters is developed. A connection between creep and 'indentation size effect' is established. (c) 2000 Elsevier Science B.V. All rights reserved.
\end{abstract}

Keywords: Hardness; Nano-indentation; Creep

\section{Introduction}

For nearly 100 years, indentation experiments have been performed to obtain the hardness of materials [1]. Recent years have seen significant improvements in indentation equipment and a growing need to measure the mechanical properties of materials on small scales. It is now possible to monitor, with high precision and accuracy, both the load and displacement of an indenter during indentation experiments [2-4]. However, questions remain, including what properties can be measured using instrumented indentation techniques and what is hardness?

Many authors have addressed these basic questions [5-18]. Rather than an exhaustive literature review, this paper summarizes our recent results [19-27], ob-

\footnotetext{
*Corresponding author. Tel.: +1-810-986-0939; fax: +1-810-9863091.

E-mail address: yang.t.cheng@gm.com (Y. Cheng).
}

tained using a scaling approach to indentation modeling, that may be useful to the interpretation of indentation hardness measurements.

We consider a three-dimensional, rigid, conical indenter of a given half angle, $\theta$, indenting normally into a homogeneous solid. The friction coefficient at the contact surface between the indenter and the solid is assumed zero. The quantities of interest from the loading portion of indentation measurements include the force $(F)$ and the contact depth $\left(h_{\mathrm{c}}\right)$ or the projected contact area $\left(A_{\mathrm{c}}\right.$; Fig. 1a), from which the hardness under load, $H=F / A_{\mathrm{c}}$, can be evaluated. In addition to the complete unloading curve, the initial unloading slope and final depth (Fig. 1b) are of particular interest for the unloading portion of indentation measurements. Furthermore, the total and reversible work of indentation, defined as the respective area under the loading and unloading curves, have also been studied. We will first review results of conical indentation into elastic-plastic solids with work-hardening $[20,26]$, followed by a new scaling theory of indentation into 


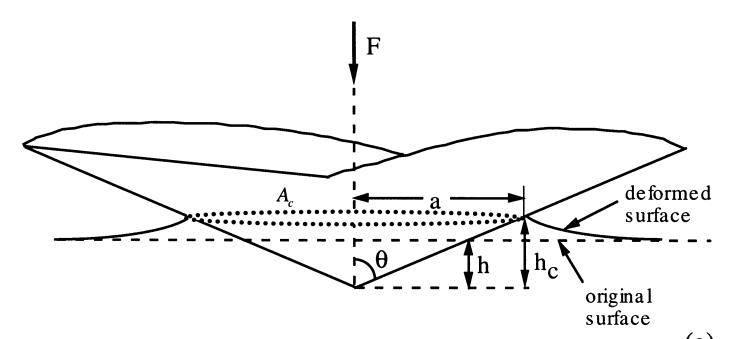

(a)

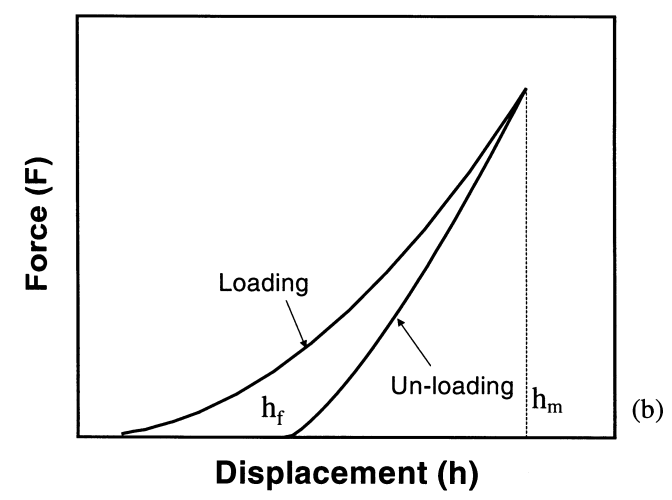

Fig. 1. Illustration of conical indentation (a) and loading-unloading curves (b).

power-law creep solid using self-similar indenters (e.g. conical and pyramidal indenters) [27].

\section{Indentation into elastic-plastic solids}

\subsection{Dimensional analysis and finite element calculations}

The stress-strain $(\sigma-\varepsilon)$ curves of the solids under uniaxial tension are assumed to be given by

$$
\begin{gathered}
\sigma=E \varepsilon \quad \text { for } \quad \varepsilon \leq \frac{Y}{E}, \\
\sigma=K \varepsilon^{n} \quad \text { for } \quad \varepsilon \geq \frac{Y}{E},
\end{gathered}
$$

where $E$ is the Young's modulus, $Y$ is the initial yield stress, $K$ is the strength coefficient, and $n$ is the work-hardening exponent [28]. To ensure continuity, we note $K=Y[E / Y]^{n}$. Consequently, either $E, Y$ and $K$ or $E, Y$ and $n$ are sufficient to describe the stress-strain relationship. We use the latter set of parameters extensively in the following discussions. When $n$ is zero, Eq. (1) becomes the model for elastic-perfectly plastic solids. For most metals $n$ has a value between 0.1 and 0.5 [29].
During loading, $F$ and $h_{\mathrm{c}}$ can be written, according to dimensional analysis $[22,25]$, as

$$
\begin{aligned}
& F=E h^{2} \Pi_{\alpha}\left(\frac{Y}{E}, v, n, \theta\right) \\
& h_{\mathrm{c}}=h \Pi_{\beta}\left(\frac{Y}{E}, v, n, \theta\right)
\end{aligned}
$$

where $\Pi_{\alpha}$ and $\Pi_{\beta}$ are dimensionless functions of four dimensionless parameters $Y / E$, (Poisson's ratio), $n$ and $\theta$. Several observations can be made. First, the force on the indenter, $F$, is proportional to the square of the indenter displacement, $h$. Second, the contact depth, $h_{\mathrm{c}}$, is proportional to the indenter displacement, $h$. Consequently, the hardness under load is independent of indenter displacement, $h$, or indenter load, $F$.

Because unloading takes place after loading during which the indenter reaches the maximum depth, $h_{\mathrm{m}}$, the equation for unloading curves is, using dimensional analysis [22,25],

$F=E h^{2} \Pi_{\gamma}\left(\frac{Y}{E}, \frac{h}{h_{\mathrm{m}}}, v, n, \theta\right)$

where $\Pi_{\gamma}$ is a dimensionless function of five parameters, $Y / E, h / h_{\mathrm{m}}, v, n$ and $\theta$. In contrast to loading, Eq. (4) shows that the force, $F$, is, in general, no longer simply proportional to the square of the indenter displacement, $h$. It also depends on the ratio, $h / h_{\mathrm{m}}$, through the dimensionless function $\Pi_{\gamma}$.

Because there is, as yet, no analytical solution to the problem of conical indentation in elastic-plastic solids, finite element calculations using ABAQUS were performed to evaluate the dimensionless functions and explore their implications [30]. For the present paper, the results for a rigid indenter of half angle of $68^{\circ}$ and a Poisson's ratio of 0.3 were summarized. To simplify notation, $\Pi_{i}(Y / E, n)(i=\alpha, \beta, \delta)$ is used instead of $\Pi_{i}\left(Y / E, 0.3, n, 68^{\circ}\right)(i=\alpha, \beta, \delta)$ in the following discussions. The calculations have since been extended to a broad range of angles from 35 to $80^{\circ}$ [31].

\subsection{Results and discussion}

\subsubsection{Indentation loading curves}

The finite element calculations confirm that the force, $F$, is indeed proportional to the square of the displacement, $h$, for conical indenter indenting a homogeneous solid with or without work-hardening. Furthermore, finite element results show [22] that a simple, approximate scaling relationship exists between $F / E h^{2}$ and $Y^{*} / E$ (Fig. 2), where $Y^{*}=(Y K)^{1 / 2}$ which may be defined as an 'effective yield strength'. Consequently, it is possible to estimate the effective yield strength, $Y^{*}$, 


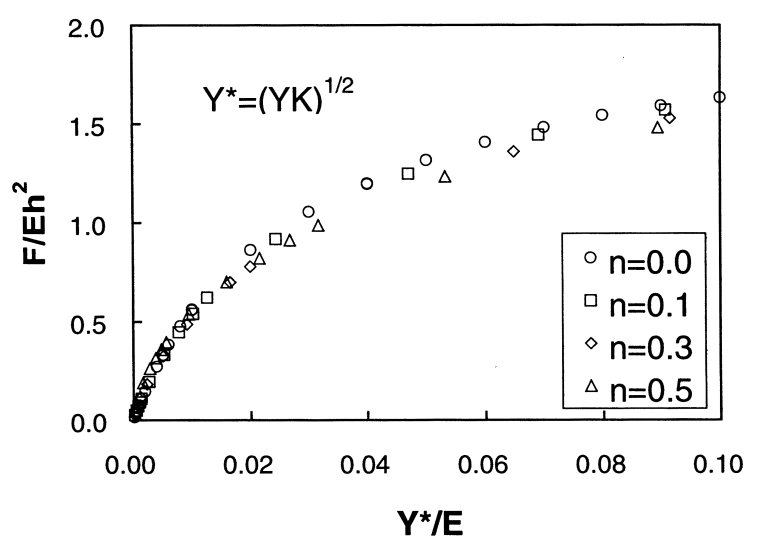

Fig. 2. An approximate scaling relationship between $F / E h^{2}$ and $Y^{*} / E$.

from indentation loading curves provided that the Young's modulus, $E$, is known. Conversely, $E$ may be obtained if $Y^{*}$ is known.

This square-dependence is characteristic of indentation, using self-similar indenters, into homogeneous solids that do not have an intrinsic length scale. Deviations from the square-dependence are expected when there is an intrinsic length associated with either the indenter or the solid. In fact, loading curves may be approximated by second-order polynomials if the indentation depth is on the same order as the tip radius of actual conical or pyramidal indenters [21]. When indenting solids that exhibit power-law creep using self-similar indenters, the loading curves can also be different from the square dependence [27] (discussed later in the paper). Consequently, the shape of indenta-
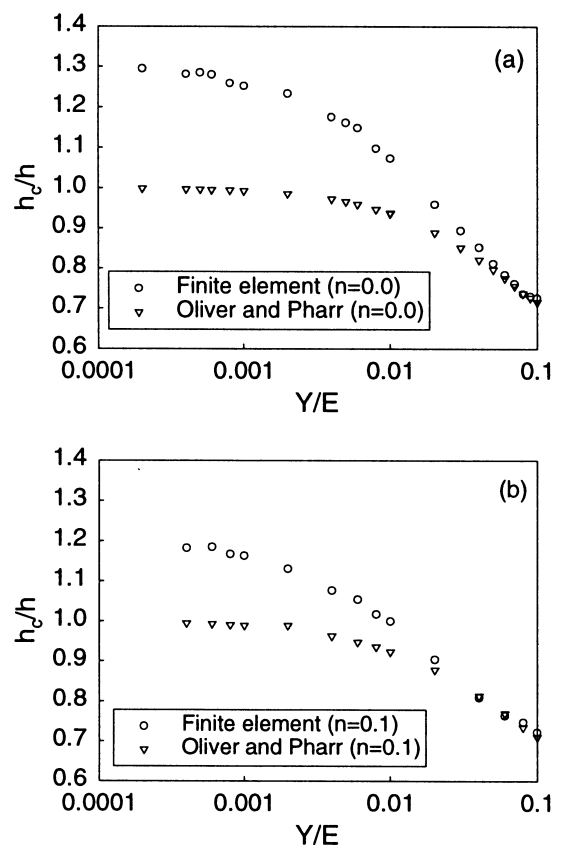

Fig. 3. Relationships between $h_{\mathrm{c}} / h$ and $Y / E$ for several values of $n$ obtained from finite element calculations and that obtained using the

Oliver-Pharr procedure for: (a) $n=0.0$; (b) $n=0.1$; (c) $n=0.3$; and (d) $n=0.5$.

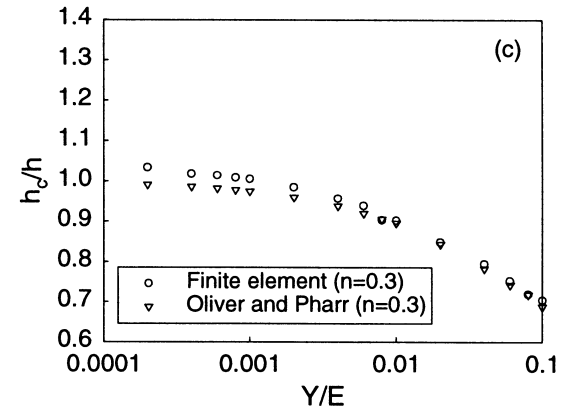

tion loading curves may be used to detect whether an intrinsic length scale exists in a material.

\subsubsection{Contact depth, sinking-in, piling-up, and a method of estimating contact depth}

The relationship between $h_{\mathrm{c}} / h$ and $Y / E$ for several values of $n$ is shown in Fig. 3a-d [23]. The value of $h_{\mathrm{c}} / h$ can be either greater or smaller than one, corresponding to the 'piling-up' and 'sinking-in' of the displaced surface profiles, respectively. For large $Y / E$, sinking-in occurs for all values of $n>0$. For small $Y / E$, both sinking-in and piling-up may occur depending on the degree of work-hardening. In the case of severe work-hardening (i.e. $n=0.5$ ), sinking-in is expected even for very small values of $Y / E$, whereas piling-up is expected for elastic-perfectly plastic solids and for solids with a small work-hardening exponent (e.g. $n=0.1$ ).

Sinking-in and piling-up of the surface profiles can cause difficulties in estimating contact depth or area. To overcome such difficulties, Oliver and Pharr have proposed a procedure for estimating contact depth from initial unloading slope [8],

$h_{\mathrm{c}}=h_{\mathrm{m}}-\xi F_{\mathrm{m}} /(\mathrm{d} F / \mathrm{d} h)_{\mathrm{m}}$

where $F_{\mathrm{m}}$ and $(\mathrm{d} F / \mathrm{d} h)_{\mathrm{m}}$ are the respective load and the initial slope of the unloading curve at the indenter displacement depth, $h_{\mathrm{m}}$. The numerical value of $\xi$ is 0.72 for conical indenter, 0.75 for the paraboloid of revolution, and 1.0 for flat punch.

Applying the Oliver and Pharr procedure to the

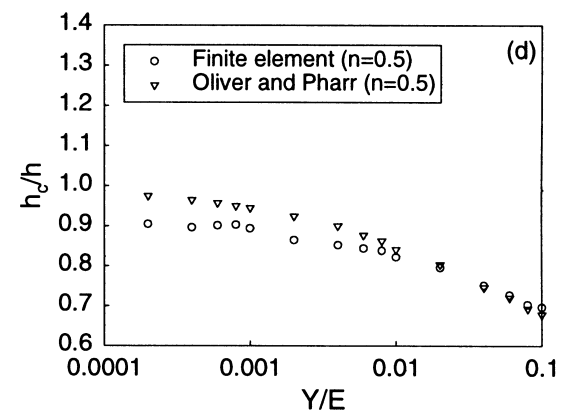


loading-unloading curves obtained from finite element calculations, we evaluate the contact depth using Eq. (5) and plot it in terms of $h_{c} / h$ in Fig. 3a-d. It is apparent from Fig. 3 that the Oliver and Pharr procedure is valid when the ratio of $Y / E$ is large (e.g. $>0.05$ for $0.0<n<0.5$ ). This is expected since this procedure is based on Sneddon's analysis of surface profiles for elastic contacts [32]. For materials with a wide range of $Y / E$ (e.g. $10^{-4}-10^{-2}$ ), such as metals, the Oliver and Pharr procedure may not be accurate. For example, the procedure underestimates the contact area for elastic-perfectly plastic solids over most $Y / E$ values (Fig. 3a). The error is most significant when piling-up occurs, i.e. $h_{\mathrm{c}} / h>1$. In fact, the contact depth, $h_{\mathrm{c}}$, estimated using Eq. (5) is always less than $h_{\mathrm{m}}$. It should also be noted that Eq. (5) could also overestimate contact area for materials with a large work-hardening exponent, e.g. $n=0.5$ (Fig. 3d). Thus, the Oliver-Pharr procedure may be used with confidence for highly elastic materials (e.g. $Y / E>0.05$ for $0.0<n<0.5)$. For materials with a wide range of $Y / E$ (e.g. $10^{-4}-10^{-2}$ ), however, this procedure should be used with caution.

\subsubsection{Relationship between hardness and mechanical properties}

Using Eqs. (2) and (3), the ratio of hardness to initial yield strength is given by [22]

$$
\frac{H}{Y}=\Pi_{h}\left(\frac{Y}{E}, v, n, \theta\right)
$$

where $\Pi_{h}$ is a dimensionless function. Clearly, the hardness is independent of the depth of indentation, $h$. The ratio $H / Y$ is, in principle, a function of $Y / E, v, n$, as well as indenter geometry $(\theta)$. Taking $\theta=68^{\circ}$ and $v=0.3$, for example, the dependence of $H / Y$ on $Y / E$ and $n$ is illustrated in Fig. 4. It is apparent that, over the practically relevant range of $Y / E$, the ratio $H / Y$ is not a constant. The hardness, $H$, depends on $Y / E$ and $n$. As expected, work-hardening has a greater effect on the hardness value for small ratio of $Y / E$. For small ratios of $Y / E$, the hardness value can be many times that of the initial yield strength, $Y$. For a large ratio of $Y / E$, the hardness value approaches 1.7 times the initial yield strength, $Y$, and is insensitive to $n$.

Tabor [1] introduced the concept of 'representative yield stress', $Y_{\mathrm{o}}$, and showed that, for conical indentation in metals, the hardness value is approximately three times $Y_{\mathrm{o}}$, where $Y_{\mathrm{o}}$ is the yield stress at a representative strain, $\varepsilon_{0}$, of $8-10 \%$. Following Tabor's idea, we evaluated $H /\left(K \varepsilon_{\mathrm{o}}^{n}\right)$ and noted an approximate scaling relationship between $H /\left(K \varepsilon_{\mathrm{o}}^{n}\right)$ and $Y / E$, if the value for strain, $\varepsilon_{0}$, is taken to be $10 \%$. In Fig. 5, we plot $\mathrm{H} /\left(0.10^{n} K\right)$ against $Y / E$. All the data points shown in Fig. 4 lie approximately on a single curve.

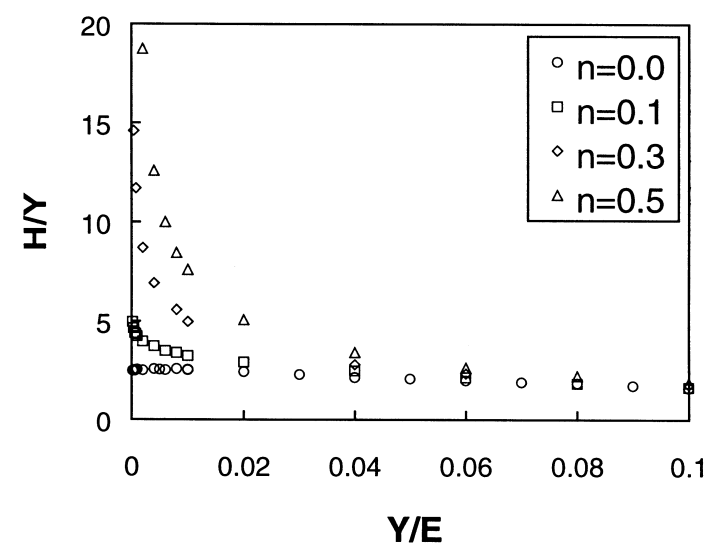

Fig. 4. Relationships between $H / Y$ and $Y / E$ for several values of $n$.

Thus, the concept of representative strain seems applicable.

It is also evident that $H /\left(K \varepsilon_{\mathrm{o}}^{n}\right)$ is a function of $Y / E$ and is, therefore, not a constant over the wide range of $Y / E$ (Fig. 5). For $Y / E<0.02, H /\left(K \varepsilon_{0}^{n}\right)$ is approximately 2.4-2.8 (Fig. 5). For $Y / E>0.06, H / Y$ is approximately $1.7-2.8$ (Fig. 4), i.e.

$H=2.8 Y_{\mathrm{o}}, \quad$ for $\quad Y / E \rightarrow 0.0$

where $Y_{\mathrm{o}}$ is the yield stress at $10 \%$ of strain, and

$H=1.7 Y, \quad$ for $\quad Y / E \rightarrow 0.1$.

2.2.4. Relationship between hardness, elastic modulus, and the work of indentation

The total work done by the indenter, $W_{\text {tot }}$, to cause elastic and plastic deformation when the indenter reaches a maximum depth and the work done by the solid to the indenter during unloading, $W_{\mathrm{u}}$, have been examined $[24,26]$. It was found that a remarkable correlation exists between the ratio of irreversible work to

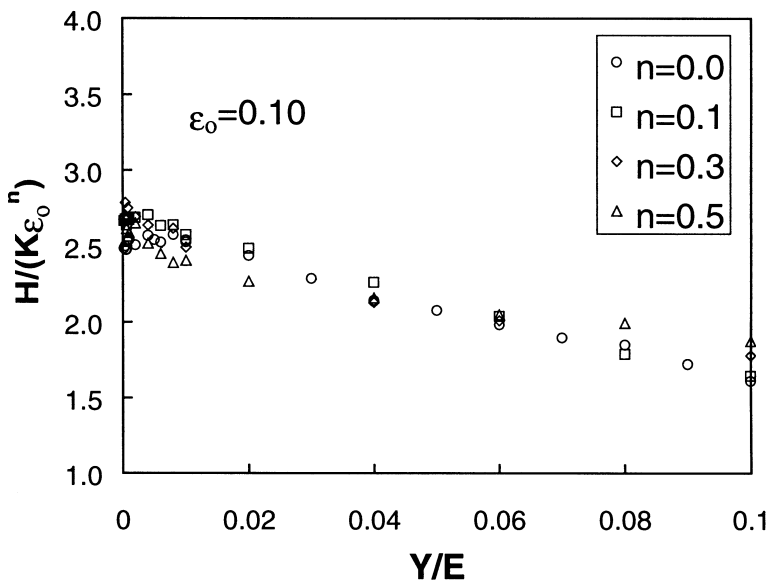

Fig. 5. A relationship between $H /\left(K \varepsilon_{\mathrm{o}}^{n}\right)$ and $Y / E$ by assuming $\varepsilon_{\mathrm{o}}=0.10$. 
total work for a complete loading-unloading cycle, $\left(W_{\text {tot }}-W_{\mathrm{u}}\right) / W_{\text {tot }}$, and the ratio of hardness to elastic modulus [24]. This correlation is illustrated in Fig. 6,

$$
\frac{H}{E^{*}} \approx \Pi_{\theta}\left(\frac{W_{\text {tot }}-W_{\mathrm{u}}}{W_{\text {tot }}}\right)
$$

where $E^{*}=E /\left(1-v^{2}\right)$. The subscript, $\theta$, denotes a possible dependence on indenter angle, since Eq. (7) and Fig. 6 were obtained for a particular indenter angle. Nevertheless, Eq. (7) shows that, for a given indenter angle, there is an approximate linear relationship between $H / E^{*}$ and $\left(W_{\text {tot }}-W_{\mathrm{u}}\right) / W_{\text {tot }}$. Consequently, the value $H / E^{*}$ may be obtained from the measurement of $W_{\mathrm{u}}$ and $W_{\text {tot }}$, which can be determined readily by simple numerical integration based on force and displacement measurements.

The ratio of hardness to elastic modulus, $H / E^{*}$, is of significant interest in tribology. This ratio multiplied by a geometric factor is the 'plasticity index' which describes the deformation properties of rough surfaces [33]. The correlation provides an alternative method for measuring $H / E^{*}$ on micro- and nano-meter scale for both metals and ceramics. Furthermore, both $H$ and $E^{*}$ may be obtained using the above correlation together with a well-known relationship between elastic modulus, contact area, and initial unloading slope $[19,24]$.

\section{Indentation into power-law creep solids}

\subsection{Dimensional analysis}

We consider a three-dimensional, rigid, conical indenter indenting normally into a homogeneous solid with power-law creep [28,29],

$\sigma=b \dot{\varepsilon}^{m}$

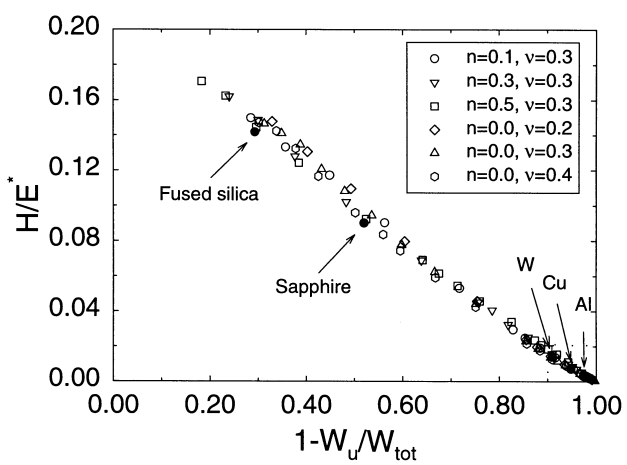

Fig. 6. A relationship between $H / E^{*}$ and $\left(W_{\text {tot }}-W_{\mathrm{u}}\right) / W_{\text {tot }}$, including data from finite element calculations for conical indenters and experimental results for a few materials using pyramidal indenters. where $\sigma$ is stress, $\dot{\varepsilon}$ is strain rate, $b$ and $m$ are material constants. For an isotropic solid obeying the creep rule given by Eq. (8), the two variables force, $F$, and contact area, $A_{\mathrm{c}}$, during loading are functions of all the independent governing parameters, $b, m$, indenter displacement $(h)$, rate of indenter displacement $(\dot{h})$, and indenter angle $(\theta)$. They are also implicitly dependent on time, $t$, since $h$ and $\dot{h}$ are dependent on time and $t=\int_{h(0)=0}^{h(t)} \mathrm{d} h / \dot{h}$. Dimensional analysis shows that [27]

$F=b\left(\frac{\dot{h}}{h}\right)^{m} h^{2} \Pi_{\alpha}^{c}(m, \theta)$

$A_{\mathrm{c}}=h^{2} \Pi_{\beta}^{c}(m, \theta)$

where $\Pi_{\alpha}^{c}$ and $\Pi_{\beta}^{c}$ are dimensionless functions of dimensionless parameters $m$ and $\theta$. Consequently, the hardness is

$H=\frac{F}{A_{c}}=b\left(\frac{\dot{h}}{h}\right)^{m} \frac{\Pi_{\alpha}^{c}}{\Pi_{\beta}^{c}} \equiv\left(b \Pi_{\gamma}^{c}\right)\left(\frac{\dot{h}}{h}\right)^{m}$

where $\Pi_{\gamma}^{c} \equiv \Pi_{\alpha}^{c} / \Pi_{\alpha}^{c}$. To simplify notation, $\Pi_{i}^{c} \equiv \Pi_{i}^{c}$ $(m, \theta)$ for $i=\alpha, \beta, \gamma$ in the following.

This equation shows that the strain rate dependence of hardness is contained in the parameter, $\dot{h} / h$. Comparing Eq. (11) with Eq. (8), we observe that, aside from the pre-factor, the power-law dependence of hardness, $H$, on $\dot{h} / h$ in indentation experiments is the same as that of stress, $\sigma$, on strain-rate, $\dot{\varepsilon}$, in uniaxial creep tests. Thus, the parameter, $\dot{h} / h$, can indeed be chosen, aside from a time-independent pre-factor, to represent indentation strain rate, as has been assumed by several authors in the past [34-40].

When the force, instead of displacement, is the independent variable, Eq. (9) may be integrated to obtain [27]:

$h(t)=\left(\frac{2}{m}\right)^{m / 2}\left(b \Pi_{\alpha}^{c}\right)^{-1 / 2}\left[\int_{0}^{t} F^{1 / m}(t) \mathrm{d} t\right]^{m / 2}$

with initial condition $h(0)=0$. In the following, the above equations are applied to several types of frequently encountered indentation experiments in which either $\dot{h}, \dot{F}$, or $\dot{F} / F$ is kept constant [34-40].

\section{Results and discussion}

4.1. Constant displacement rate, $\dot{h}=\dot{h}_{c}$, experiments

When $\dot{h}_{c}$ is constant, the force and hardness are, according to Eqs. (9) and (11), 
$F=\left(b \Pi_{\alpha}^{c}\right)\left(\frac{\dot{h}_{c}}{h}\right)^{m} h^{2}$

$H=\left(b \Pi_{\gamma}^{c}\right)\left(\frac{\dot{h}_{c}}{h}\right)^{m}$

Thus, the force during loading is proportional to $h^{2-m}$ and is no longer proportional to $h^{2}$. The hardness decreases with indentation depth. The creep exponent, $m$, can be obtained from either the indentation loading curve or from the graph of $\ln (H)$ vs. $\ln (\dot{h} / h)$.

\subsection{Constant loading rate, $\dot{F}=\dot{F}_{c}$, experiments}

When $\dot{F}_{c}$ is constant, it can be shown that the force and hardness are [27],

$F=\left(b \Pi_{\alpha}^{c}\right)^{1 /(m+1)}\left[\dot{F}_{c} \frac{m+1}{2}\right]^{m /(m+1)} h^{2 /(m+1)}$

$H=\left(b \Pi_{\gamma}^{c}\right)\left(\frac{\dot{h}}{h}\right)^{m}=\left(b \Pi_{\gamma}^{c}\right)\left(\frac{m+1}{2}\right)^{m}\left(\frac{\dot{F}_{c}}{F}\right)^{m}$

Thus, the force during loading is proportional to $h^{2 /(m+1)}$. The hardness decreases with increasing indentation load. The creep exponent, $m$, can be obtained from either the indentation loading curve, the graph of $\ln (H)$ vs. $\ln (\dot{h} / h)$, or the graph of $\ln (H)$ vs. $\ln (\dot{F} / F)$.

\subsection{Constant loading rate over load, $\dot{F} / F$, experiments}

Since $(\dot{F} / F)=\lambda$ is a constant, the force is given by $F=F_{0} \mathrm{e}^{\lambda t}$, where $F_{0}$ is the force at $t=0$. Substituting into Eq. (12), we obtain a solution:

$h(t)=\frac{1}{\sqrt{b \Pi_{\alpha}^{c}}}\left(\frac{2}{\lambda}\right)^{m / 2} F_{0}^{1 / 2}\left(\mathrm{e}^{\lambda t / m}-1\right)^{m / 2}$

and for large $t>m / \lambda$,

$h(t) \approx\left(\frac{2^{m} F_{0}}{\lambda^{m} b \Pi_{\alpha}^{c}}\right)^{1 / 2} \mathrm{e}^{\lambda t / 2}$

Consequently, the indentation strain rate is given by

$\frac{\dot{h}}{h}=\frac{\lambda}{2}\left(1-\mathrm{e}^{-\lambda t / m}\right)^{-1} \approx \frac{\lambda}{2}=\frac{1}{2} \frac{\dot{F}}{F}$

Thus, the indentation strain-rate, $\dot{h} / h$ is half of $\dot{F} / F$ after a transient period of the order of $m / \lambda$. Using
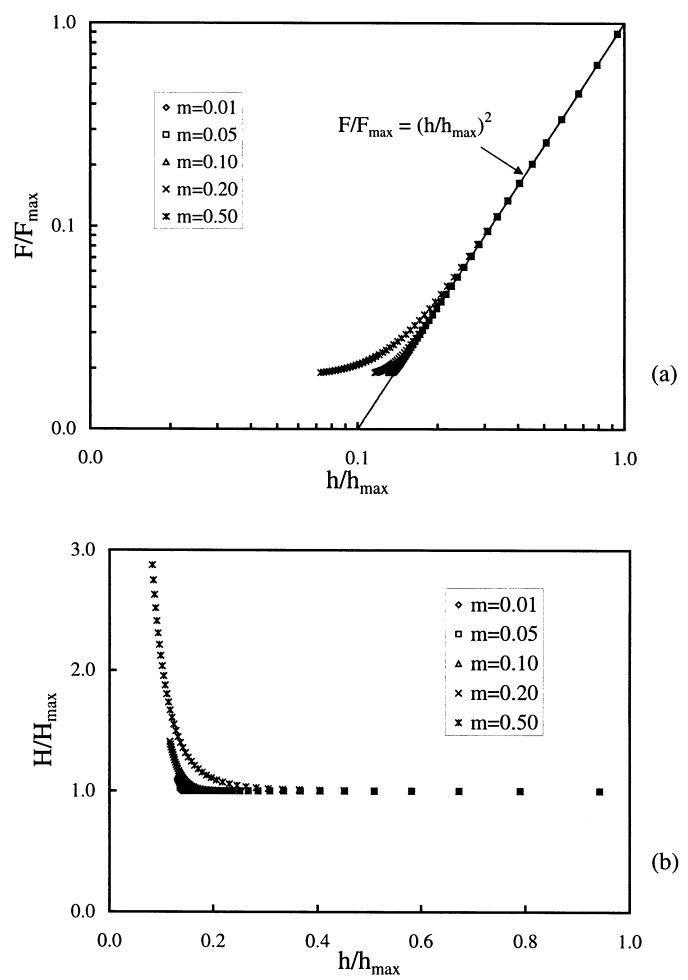

Fig. 7. Scaling relationships between indenter load and displacement (a) and between hardness and indenter displacement (b) for constant loading rate over load cases where the parameter $\lambda t_{\max }=3$.

Eqs. (9) and (11), the respective indentation loading curve and hardness may be written,

$F=F_{0} \mathrm{e}^{\lambda t} \approx\left(b \Pi_{\alpha}^{c}\right)\left(\frac{\lambda}{2}\right)^{m} h^{2}$

$H=\left(b \Pi_{\gamma}^{c}\right)\left(\frac{\dot{h}}{h}\right)^{m} \approx\left(b \Pi_{\gamma}^{c}\right)\left(\frac{\lambda}{2}\right)^{m}$

Eqs. (20) and (21), scaled by their respective values at $h_{m}$, are shown in Fig. 7. Clearly, hardness reaches a steady state value when $\dot{F} / F$ is kept constant. Correspondingly, the loading force is again proportional to $h^{2}$. The hardness increases with $(\dot{F} / F)^{m}$. The creep exponent, $m$, can be obtained from either the indentation loading curve, the graph of $\ln (H)$ vs. $\ln (\dot{F} / F)$, or of $\ln (H)$ vs. $\ln (\dot{h} / h)$.

The results of the above analysis are consistent with experimental data from the literature. For example, numerous authors have shown a linear dependence between $\ln (H)$ and $\ln (\dot{h} / h)$ for all three loading conditions considered above (i.e. either $\dot{h}, \dot{F}$, or $\dot{F} / F$ is kept constant) [34-40]. Furthermore, the creep exponent, $m$, has been obtained from the slope of the straight lines in the graph of $\ln (H)$ vs. $\ln (\dot{h} / h)$ [34-40]. The creep exponent, $m$, has also been obtained from 
indentation loading curves by Grau et al. using either constant $\dot{h}$ or $\dot{F}$ experiments and equations similar to Eqs. (13) and (15) [39].

Several authors have reported an 'indentation size effect' in constant $\dot{h}$ or $\dot{F}$ experiments as predicted by the above equations [Eqs. (14) and (16)]: hardness decreases with increasing indentation depth or load $[37,40]$. It has also been demonstrated recently by Lucas and Oliver [40] that in constant $\dot{F} / F$ experiments, the indentation strain rate reaches a 'steady state' and is given by $0.5 \dot{F} / F$ in agreement with Eq. (19). Furthermore, these authors showed [40] that the steady state hardness is independent of indentation depth and is proportional to $(\dot{F} / F)^{m}$ as predicted by Eq. (21).

\section{Conclusions}

We have derived scaling relationships for loading and unloading curve, contact depth, and hardness for indentation in elastic-plastic solids with work-hardening. The square dependence of loading curves is characteristic of indentation in homogenous solids using self-similar indenters (e.g. conical and pyramidal). For a given indenter geometry, hardness depends on both the elastic and plastic properties of materials and is not necessarily three times the yield strength. The conditions for 'piling-up' and 'sinking-in' of surface profiles during indentation were obtained. The Oliver-Pharr procedure for estimating contact depth may be used with confidence for highly elastic materials. However, this procedure may cause significant error when pilingup occurs. A relationship between the ratio of hardness to elastic modulus and the ratio of irreversible work to total work was found. This relationship offers a new method for obtaining hardness and elastic modulus. Finally, a scaling theory for indentation in power-law creep solids using self-similar indenters was developed. An 'indentation size effect' is expected in experiments using either constant displacement rate or constant loading rate. In contrast, hardness reaches a steady state value in experiments using constant loading rate over load.

These conclusions are the results of the scaling theory based on several clearly specified assumptions, including the ones that describe the behavior of materials [e.g. Eqs. (1) and (8)]. In reality, however, materials behavior may be much more complex. For hard materials, for example, the deformation mechanism responsible for the hardness impression may include a significant fracture component [41] instead of the purely elastic-plastic behavior given by Eq. (1). Likewise, alternative mechanisms may be responsible for the observed indentation size effects, including straingradient plasticity [42], imperfection in the indenter geometry [43], and surface roughness [44]. Neverthe- less, the conclusions of this study provide a framework for understanding indentation hardness measurements for two classes of materials, i.e. elastic-plastic solids with power-law work-hardening [Eq. (1)] and solids with power-law creep [Eq. (8)]. These results of this work may also be used to identify new mechanisms responsible for deformation in indentation experiments.

\section{Acknowledgements}

We would like to thank Z. Li, W.J. Meng, S.J. Harris, G.L. Eesley, J.R. Smith, K.C. Taylor for helpful discussions. Correspondences with Professors D. Tabor, G.M. Pharr, and W.D. Nix have also been valuable.

\section{References}

[1] D. Tabor, The Hardness of Metals, Oxford, London, 1951; Phil. Mag. A74 (1996) 1207.

[2] J.B. Pethica, R. Hutchings, W.C. Oliver, Phil. Mag. A48 (1983) 593.

[3] D. Stone, W.R. LaFontaine, P. Alexopoulos, T.W. Wu, C.-Y. Li, J. Mater. Res. 3 (1988) 141.

[4] B. Bhushan, A. Kulkarni, W. Bonin, J. Wyrobek, Phil. Mag. A74 (1996) 1117.

[5] M.F. Doerner, W.D. Nix, J. Mater. Res. 1 (1986) 601.

[6] J.L. Loubet, J.M. Georges, G. Meille, in: P.J. Blau, B.R. Lawn (Eds.), Microindentation Techniques in Materials Science and Engineering, ASTM STP 889, American Society for Testing and Materials, Philadelphia, 1986, p. 72.

[7] A.K. Bhattacharya, W.D. Nix, Int. J. Solids Struct. 24 (1988) 881.

[8] W.C. Oliver, G.M. Pharr, J. Mater. Res. 7 (1992) 1564.

[9] E.R. Kral, K. Komvopoulos, D.B. Bogy, J. Appl. Mech. 60 (1993) 829.

[10] S.A. Syed Asif, B. Derby, S.G. Roberts, Mater. Res. Soc. Symp. Proc. 356 (1995) 681.

[11] A. Bolshakov, W.C. Oliver, G.M. Pharr, J. Mater. Res. 11 (1996) 760.

[12] S.V. Hainsworth, H.W. Chandler, T.F. Page, J. Mater. Res. 11 (1987) 1996.

[13] B. Rother, Surf. Coat. Technol. 86/87 (1996) 535.

[14] A. Faulkner, K.C. Tang, S. Sen, R.D. Arnell, J. Strain Anal. 33 (1998) 411.

[15] J.A. Knapp, D.M. Follstaedt, S.M. Myers, J.C. Barbour, T.A. Friedmann, J. Appl. Phys. 85 (1999) 1460.

[16] M. Sakai, Acta Metall. Mater. 41 (1993) 1751; J. Mater. Res. 14 (1999) 3630.

[17] A.E. Giannakopoulos, S. Suresh, Scr. Mater. 40 (1999) 1191.

[18] Y. Sun, S. Zheng, T. Bell, J. Smith, Phil. Mag. Lett. 79 (1999) 649.

[19] C.-M. Cheng, Y.-T. Cheng, Appl. Phys. Lett. 71 (1997) 2623.

[20] Y.-T. Cheng, C.-M. Cheng, Phil. Mag. Lett. 77 (1998) 39.

[21] Y.-T. Cheng, C.-M. Cheng, J. Mater. Res. 13 (1998) 1059.

[22] Y.-T. Cheng, C.-M. Cheng, J. Appl. Phys. 84 (1998) 1284.

[23] Y.-T. Cheng, C.-M. Cheng, Phil. Mag. Lett. 78 (1998) 115.

[24] Y.-T. Cheng, C.-M. Cheng, Appl. Phys. Lett. 73 (1998) 614.

[25] Y.-T. Cheng, C.-M. Cheng, Int. J. Solids Struct. 36 (1999) 1231.

[26] Y.-T. Cheng, C.-M. Cheng, J. Mater. Res. Rapid Commun. 14 (1999) 3493.

[27] Y.-T. Cheng, C.-M. Cheng, A Scaling Theory of Indentation in 
Power-Law Creep Solids using Self-Similar Indenters, GM R \& D Publication R\& D-8994, 1999.

[28] J. Lubliner, Plasticity Theory, Macmillan, New York, 1990.

[29] G. Dieter, Mechanical Metallurgy, 2nd ed, McGraw-Hill, New York, 1976.

[30] ABAQUS, version 5.6, Hibbitt, Karlsson \& Sorensen, Inc., Pawtucket, RI 02860, USA.

[31] Z. Li, Y.-T. Cheng (to be published).

[32] I.N. Sneddon, Int. J. Eng. Sci. 3 (1963) 47.

[33] J.A. Greenwood, J.B.P. Williamson, Proc. R. Soc. A295 (1966) 300.

[34] A.G. Atkins, A. Silverio, D. Tabor, J. Inst. Met. 94 (1966) 369.

[35] M.J. Mayo, W.D. Nix, Acta Metall. Mater. 36 (1988) 2183.
[36] W.R. LaFontaine, B. Yost, R.D. Black, C.-Y. Li, J. Mater. Res. 5 (1990) 2100.

[37] D.S. Stone, K.B. Yoder, J. Mater. Res. 9 (1994) 2524.

[38] S.A. Syed, J.B. Pethica, Phil. Mag. A 76 (1997) 1105.

[39] P. Grau, G. Berg, H. Meinhard, S. Mosch, J. Am. Ceram. Soc. 81 (1998) 1557.

[40] B.N. Lucas, W.C. Oliver, Metall. Mater. Trans. 30A (1999) 601. [41] I.J. McColm, Ceramic Hardness, Plenum, New York, 1990.

[42] H. Gao, Y. Huang, W.D. Nix, J.W. Hutchinson, J. Mech. Phys. Solids 47 (1999) 1239.

[43] A. Iost, R. Bigot, J. Mater. Sci. 31 (1996) 3573.

[44] M.S. Bobji, S.K. Biswas, Tribol. Lett. 7 (1999) 51. 